\title{
Edge Computing with ParaDrop Tutorial
}

\author{
Suman Banerjee \\ Department of Computer Science \\ University of Wisconsin-Madison \\ Madison, WI, USA \\ suman@cs.wisc.edu
}

\begin{abstract}
This half-day tutorial will explore edge computing through hands-on development activities using a physical edge computing platform. With the recent trends in the networking ecosystem-the rise of IoT devices, high bandwidth wireless, and powerful, energy-efficient, and inexpensive computation-edge computing is a promising technology for highly interactive and immersive environments. This tutorial aims to promote awareness of the possibilities of edge computing in general and introduce attendees to the tools that they can use to begin exploring this space. The tutorial's activities will give attendees hands-on experience with the ParaDrop edge computing platform developed at the University of Wisconsin-Madison. We hope to impart working knowledge about edge computing and our vision for its future.
\end{abstract}

Recent developments in home wireless networks such as the proliferation of connected devices (e.g. Internet-ofThings) and gigabit wireless (e.g. 802.11ac) create an environment where edge computing can truly enhance applications. The availability of low latency and high bandwidth at the network edge but not necessarily end-toend to the cloud, can enable interesting new applications involving video (e.g. augmented reality), low latency sensor-actuator coordination, and other public safety or educational applications. However, before such applications emerge, there needs to be a platform available for them.

We argue that the availability of widespread platforms and standards for edge computing will unleash a new wave of innovation much like the creation of app markets for mobile devices. The hardware exists, e.g. in the form of millions of always-on Wi-Fi routers in homes and businesses, but it is not very programmable. With our tutorial, we aim to foster awareness of the possibilities

Permission to make digital or hard copies of part or all of this work for personal or classroom use is granted without fee provided that copies are not made or distributed for profit or commercial advantage and that copies bear this notice and the full citation on the first page. Copyrights for third-party components of this work must be honored. For all other uses, contact the Owner/Author.

MobiCom'18, October 29-November 2, 2018, New Delhi, India.

(c) 2018 Copyright is held by the owner/author(s).

ACM ISBN 978-1-4503-5903-0/18/10.

DOI: https://doi.org/10.1145/3241539.3270097 that exist with edge computing and promote the advancement of research toward good standards for its realization. We introduce the ParaDrop platform as an open source demonstration of our vision for edge computing. We have been single-mindedly focused on researcher and developer needs in creating the ParaDrop platform. As such, applications that are already written to be run as cloud services can be easily modified to run on the ParaDrop platform in order to benefit from running at the network edge. Applications are not required to be written in a highly specialized language, and applications are able to leverage the rapidly growing software ecosystem surrounding Docker. Our tutorial will give researchers a hands-on experience with the platform and knowledge that they will be able to use beyond the workshop.

\section{CCS Concepts/ACM Classifiers}

• General and reference General literature • Networks

\section{Author Keywords \\ Edge Computing}

\section{BIOGRAPHY}

Suman Banerjee is a Professor in $\mathrm{CS}$ at UW-Madison where he is the founding director of the WiNGS laboratory, focusing on research in wireless and mobile networking systems, with broader interests in networking and distributed systems. $\mathrm{He}$ received his undergraduate

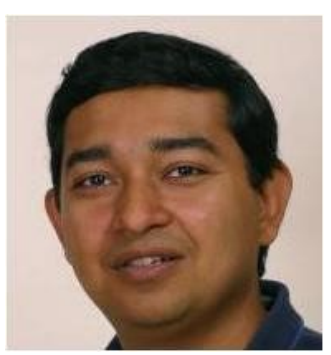
degree from IIT Kanpur, and MS and $\mathrm{PhD}$ degrees from the University of Maryland. He is the inaugural recipient of the ACM SIGMOBILE Rockstar award and a recipient of the NSF Career Award. He is a recipient of multiple award papers at various conferences, such as ACM MobiCom, ACM CoNEXT, and IEEE Dyspan. 